\title{
PREDICTING THE RANGE OF INBRED LINES
}

\author{
J. L. JINKS and JEAN M. PERKINS \\ Department of Genetics, University of Birmingham, \\ Birmingham B15 2TT
}

Received 12.i.72

\section{THEORY}

Although the prediction of the expected response to selection from estimates of environmental and genetical parameters obtained from the base population has attracted considerable theoretical and practical attention, there have been no corresponding developments in predicting the properties of the extreme phenotypes that are expected to emerge since the initial step was taken by Mather (1949). Nevertheless, such predictions would have at least the same practical and theoretical interest. For the simplest case, where the base population is the $\mathrm{F}_{2}$ of a cross between two pure breeding lines and the genes are independent in their action and in their segregation, i.e. no epistasis and no linkage, the theoretical basis of such predictions follows directly from the treatment of continuously varying characters of Mather and Jinks (1971). And, as we shall see, the experimental observations required to make the predictions are relatively undemanding.

Following Mather and Jinks (1971), we can define the means of any pair of pure breeding lines around their joint mean, $m$, as

and

$$
\begin{aligned}
& \bar{P}_{1}=m+r_{d} \Sigma d_{i} \\
& \bar{P}_{2}=m-r_{d} \Sigma d_{i},
\end{aligned}
$$

where $r_{d}$ is the coefficient of association of genes of like effect, $\left(r_{d}=1\right.$ to 0$)$ $d_{i}$ is the additive effect of the $i$ th locus and the summation is over the $k$ loci $\left(i=1\right.$ to $k$ ) at which $P_{1}$ and $P_{2}$ differ.

The mean of the $F_{1}$ obtained on crossing this pair of lines is then

$$
F_{1}=m+\Sigma h_{i},
$$

where $h_{i}$ is the dominance effect of the $i$ th locus $(i=1$ to $k)$ and

$$
\Sigma h_{i}=F_{1}-\frac{1}{2}\left(\bar{P}_{1}+\bar{P}_{2}\right) \text {. }
$$

The means of the highest $\left(P_{H}\right)$ and lowest $\left(P_{L}\right)$ scoring inbred lines that could emerge from this cross following many generations of inbreeding are then

and

$$
\begin{aligned}
& \bar{P}_{H}=m+\Sigma d_{i} \\
& \bar{P}_{L}=m-\Sigma d_{i}, \text { respectively }
\end{aligned}
$$

The genetical components of variation which can be estimated from observations on the variation within and between families of appropriate segregating generations are

and

$$
\begin{aligned}
& D=\Sigma d_{i}^{2} \\
& H=\Sigma h_{i}^{2} \\
& F={ }_{399} d_{i} h_{i} .
\end{aligned}
$$


If $h_{i} \mid d_{i}$ is a constant for all loci, i.e. $h_{i}=f d_{i}$ then,

$$
\Sigma h_{i} \sqrt{\frac{D}{H}}=\Sigma f d_{i} \sqrt{\frac{\Sigma d_{i}^{2}}{\Sigma f^{2} d_{i}^{2}}}=\Sigma d_{i}
$$

from which we can immediately estimate $\bar{P}_{H}$ and $\bar{P}_{L}$ as $m \pm \Sigma d_{i}$.

If $h_{i}=f d_{i}$ the ratio $F / \sqrt{D . H}$ equals one providing that all dominant alleles are present in one of the two parents $\left(P_{1}\right.$ or $\left.P_{2}\right)$ and all recessive alleles in the other. If we observe a value of one for this ratio it therefore shows that the assumption of a constant dominance ratio over all loci is valid. However, a value of less than 1 , or even 0 , is compatible with a constant ratio over all loci if the dominant alleles are equally divided between $P_{1}$ and $P_{2}$. We can, therefore, estimate the highest and lowest scoring inbred lines by assuming a constant dominance ratio over all loci and we have a method of proving that this assumption holds in some circumstances. This procedure does not require an estimate of $k$ in order to estimate $\bar{P}_{H}$ and $\bar{P}_{L}$ : it thus avoids this limiting factor in Mather's earlier approach (Mather, 1949).

\section{Practice}

An experimental design that produces reliable estimates of all the parameters necessary to make these predictions and at the same time provides tests of the underlying assumptions has been described by Kearsey and Jinks (1968) and Jinks and Perkins (1970). In this design the $F_{2}$ of a cross between two pure-breeding lines $\left(\mathbf{P}_{1}\right.$ and $\left.\mathbf{P}_{2}\right)$ is back-crossed to these lines and to their $F_{1}$. The resulting families are raised simultaneously with the pure breeding lines, their $F_{1}, F_{2}$ and backcrosses of the $F_{1}$ to $P_{1}$ and $P_{2}\left(B_{1}\right.$ and $\mathrm{B}_{2}$, respectively). An analysis of variance provides tests of significance of the additive, dominance and epistatic components of variation and in the absence of the latter orthogonal estimates of $D, H$ and $F$. A joint scaling test on the means of the $\mathrm{P}_{1}, \mathrm{P}_{2}, \mathrm{~F}_{1}, \mathrm{~F}_{2}, \mathrm{~B}_{1}$ and $\mathrm{B}_{2}$ families provides a further test for the presence of epistasis and in its absence weighted least squares estimates of $m, r_{d} \Sigma d_{i}$ and $\Sigma h_{i}$ (Mather and Jinks, 1971). A comparison of the variances of $F_{2}$ and $F_{2} \times F_{1}, B_{1}$ and $F_{2} \times P_{1}$ and $B_{2}$ and $F_{2} \times P_{2}$ provide independent tests for the presence of linkage and of the linkage phase (Perkins and Jinks, 1970). Thus we have estimates of all relevant parameters and tests of all assumptions.

\section{Prediction}

This design has been carried out once only as a relatively small part of a much larger experiment. The cross was between varieties 1 and 5 of Nicotiana rustica (Mather and Vines, 1952). All the necessary families were grown in 1965 and final height was recorded in inches. The complete experiment, of which this was a part, has been described and analysed in a number of papers (Jinks and Perkins, 1969, 1970; Perkins and Jinks, 1970). Neither the scaling test nor the triple-test-cross gave any evidence of epistasis, although analyses of more extensive bodies of data covering many more generations show that epistasis occurs at a low level in some environ- 
ments (Jinks and Perkins, 1969). There was, however, evidence of repulsion linkage of relatively small effect but nevertheless significant because of the large number of degrees of freedom available (Perkins and Jinks, 1970, table 8). While, therefore, the initial assumption of independence of the genes is not fully met the failure is likely to have only marginal consequences The estimates of the relevant parameters are

$$
\begin{aligned}
\Sigma \hat{h}_{i} & =6.11 \\
\hat{D} & =30.69 \\
\hat{H} & =4.08 \\
\hat{F} & =0.41 .
\end{aligned}
$$

The estimate of $F$ is not significantly different from zero and the ratio $F / \sqrt{D . H}$ is clearly less than one. Hence we have no proof that $h_{i} / d_{i}$ is constant over all loci, but equally we have no evidence to the contrary. We shall proceed therefore on the assumption that the dominance ratio is constant. The prediction is then:

$$
\Sigma \hat{d}_{i}=6 \cdot 11 \sqrt{\frac{30 \cdot 69}{4 \cdot 08}}=16 \cdot 76 .
$$

As part of the same experiment, a second triple-test-cross was carried out on the two first back-crosses, $\mathbf{B}_{1}$ and $\mathbf{B}_{2}$. When pooled, the two backcrosses should yield the same estimates of the relevant parameters as the $\mathrm{F}_{2}$. Using the estimates from the backcross population we obtain

$$
\Sigma \hat{d}_{i}=16 \cdot 71 \text {. }
$$

Relaxing the condition that the estimates of all parameters come from the same experiment, considerably more data becomes available. Thus we have a mean estimate of $\Sigma h_{i}$ over 16 independent experiments (Bucio Alanis, Perkins and Jinks, 1969) and estimates of $D, H$ and $F$ based on the mean estimates from seven complete or incomplete triple-test-crosses (Jinks and Perkins, 1970). These estimates are:

$$
\begin{aligned}
\Sigma \hat{h}_{i} & =5.82 \\
\hat{D} & =39.98 \\
\hat{H} & =11.48 \\
\hat{F} & =-2.08
\end{aligned}
$$

from which we estimate

$$
\Sigma d_{i}=10 \cdot 86
$$

These three predictions are sufficiently close to one another for us to place some confidence in them. On the other hand, the errors of these predictions whether based on their theoretical errors or observed errors of the estimates of the parameters are between a half and a third of the magnitude of the values of $\Sigma d_{i}$.

The practical value of the predictions can, however, be tested directly in the case of the $1 \times 5$ cross. Perkins and Jinks (unpublished) produced 82 $\mathrm{F}_{8}$ families by repeated selfing from an initial random sample of $100 \mathrm{~F}_{2}$ plants of the 1965 experiment. The means of the two families that were the extremes for final height were found by our colleague R. J. Brumpton to be $61.49 \pm 1.64$ and $34.53 \pm 0.48$ inches respectively when grown in 1970 . 
These give an estimate of $\Sigma d_{i}$ equal to $13.48 \pm 0 \cdot 86$, which falls within the range of the predicted values. We can, however, make a further prediction based on the estimate of $\Sigma h_{i}$ observed in the same 1970 experiment. With the average values of $D$ and $H$ over seven experiments this gives a prediction of

$$
\Sigma \hat{d}_{i}=6 \cdot 79 \sqrt{\frac{39 \cdot 98}{11 \cdot 48}}=12 \cdot 68,
$$

which is a good fit with the observed value. Thus the predictions appear to be somewhat better than their sampling errors would suggest.

We can now use corresponding observed values of $r_{d} \Sigma d_{i}, D$ and predicted values of $\Sigma d_{i}$ to estimate $r_{d}$ and $k$. The three sets of values available give $r_{d}=0.31,0.26$ and 0.21 and $k=9.15,2.95$ and 4.50 respectively. The same estimates made from the analysis of final height in $F_{8}$ families are $r_{d}=0.22$ and $k=4$ (Eaves and Brumpton, 1972), which are in a sense realised values of the parameters. It is worth noting that the estimates of $r_{d}, k, \Sigma d_{i}$ and $\Sigma h_{i}$ leave no doubt that the heterosis observed in the $\mathrm{F}_{1}$ of this cross (Bucio Alanis, Perkins and Jinks, 1969) is due to a number of partially dominant increasing alleles dispersed between the two parents. In these circumstances we expect to be able to extract pure-breeding lines from the cross that exceed the better parent and the $F_{1}$ and we have not only done so but we have correctly predicted the mean of the best inbred line six generations in advance.

\section{FutURe DEVElopMents}

In spite of the fact that two of the assumptions underlying the predictions, namely, no epistasis and no linkage, are not fully met in the $1 \times 5$ cross predictions of the phenotypes of the extremes among the inbred lines appear to be reasonable when judged against those obtained. Only further elaboration of the theory and further experimental tests of the predictions can tell us whether or not the practical value of the predictions is as good as the $1 \times 5$ cross would suggest.

The means and variances of families derived from the cross $1 \times 5$ contain significant contributions attributable to interactions between genotypes and seasons (Bucio Alanis, Perkins and Jinks, 1969; Perkins and Jinks, 1970). Estimates of the parameters and hence of the predictions based upon them will vary with the season. And we expect and indeed observe better agreement between predictions and between predictions and observations when they are based on information from the same experiment than when they come from different experiments. On the other hand, the earlier in a breeding programme that a reliable prediction can be made, the greater is its prospective practical value. Any general treatment must, therefore, allow for the contribution of genotype-environmental interactions to the parameters used in the prediction. In general we would expect their contributions to be more critical for the estimate of $\Sigma h_{i}$ than for the ratio of $D / H$. Further developments of the theory must also remove the assumptions of no linkage and no epistatis if the method is to be more generally applicable, although with inbreeding by selfing the opportunities for recombination after the $F_{2}$ generation are so restricted that the components of variation estimated from triple-test-crosses on the $F_{2}$ are likely to be biassed in the 
right direction for predicting the range of inbred lines in later generations in the presence of linkage.

\section{Summary}

1. A method is described for predicting the range of phenotypes expected among the true-breeding lines that can be obtained by inbreeding from the $\mathrm{F}_{2}$ of a cross between a pair of inbred lines assuming independence of the genes in action and in segregation, and a constant dominance ratio over all loci.

2. A breeding programme is described which provides estimates of all the parameters required to make the predictions and to test all the underlying assumptions except the constancy of the dominance ratio over all loci for which a test is available only in restricted circumstances.

3. This prediction for final height in the cross between varieties 1 and 5 of Nicotiana rustica shows a good agreement with the results of inbreeding by selfing a random sample of $100 \mathrm{~F}_{2}$ individuals of this cross in spite of the failure of some of the assumptions.

4. The necessary elaborations of the method to make it more generally applicable are outlined.

Acknowledgments.-We are indebted to Professor K. Mather for helpful discussion.

\section{REFERENGES}

BUCIO ALANIS, L., PERKINS, JEAN M., AND JINKS, J. L. 1969. Environmental and genotypeenvironmental components of variability. V. Segregating generations. Heredity, 24, $115-127$.

EAVES, L. J., AND BRUMPTON, R. J. 1972. Factors of covariation in Nicotiana rustica. Heredity, In Press.

JINKS, J. L., AND PERKINS, JEAN M. 1969. The detection of linked epistatic genes for a metrical trait. Heredity, 24, 465-475.

JINKS, J. L., AND PERKINS, JEAN M. 1970. A general method for the detection of additive dominance and epistatic components of variation. III. $\mathrm{F}_{2}$ and backcross populations. Heredity, 25, 419-429.

KEARSEY, M. J., AND JINKs, J. L. 1968. A general method for the detection of additive, dominance and epistatic components of variation for metrical traits. I. Theory. Heredity, 23, 403-409.

MATHER, k. 1949. Biometrical Genetics. 1st ed. London: Methuen.

MATHER, K., AND JINKS, J. L. 1971. Biometrical Genetics. 2nd ed. London: Chapman Hall. MATHER, K., AND VINES, A. 1952. The inheritance of height and flowering time in a cross of Nicotiana rustica. In Quantitative Inheritance (Ed. E. C. R. Reeve and C. H. Waddington), pp. 49-80. London: H.M.S.O.

PERKINS, JEAN M., AND JINKS, J. L. 1970. Detection and estimation of genotype-environmental, linkage and epistatic components of variation for a metrical trait. Heredity, 25, 157-177. 